\title{
Note on a Test of the Equivalence Theorem for Sporadic E Propagation
}

\author{
J. W. Wright and T. N. Gautier
}

(February 4, 1960; revised February 23, 1960)

\begin{abstract}
Analysis of two days (123 cases) of sporadic $E$ observed simultaneously at oblique and vertical incidence verifies that the classical sec $\Phi$ relationship between top frequencies is roughly appropriate for sporadic $E$.
\end{abstract}

\section{Introduction}

Medium distance radio propagation (up to 2,000 $\mathrm{km})$ is frequently enhanced through reflection from the sporadic $E$ layer $(E \mathrm{~s})$. At vertical incidence, reflections from $E$ s appear to be essentially specular, in the sense that little or no gradual refraction takes place within the layer; this is in contrast to "reflection" from the regular $E$ and $F$ layers, in which a more gradual refraction process is involved. On the other hand, reflections from $E$ s are often observed to be only partial, and the nature of the reflecting surface and, therefore, the reflecting mechanism, are still in some doubt. (See Thomas and Smith [1] ${ }^{2}$ for a review of current knowledge of $E_{\mathrm{s}}$.) Thus the relationship of vertical-incidence $E$ s measurements to oblique-incidence $E$ s propagation is also uncertain from a theoretical point of view.

For the simple case of a plane, parallel, earth, and ionosphere, and no magnetic field, the classical "secant law" states that

$$
f_{\mathrm{ob} 1}=f_{v} \sec \Phi,
$$

where $f_{\mathrm{ob} 1}$ and $f_{v}$ are the oblique and vertical incidence frequencies of reflection, respectively, from a plasma frequency $f_{p}=f_{v}$, and $\Phi$ is the angle of incidence of $f_{\mathrm{ob} 1}$ on the layer.

This relation is applicable not only to radio reflections from a horizontally stratified layer but also to scattering by a stratum of ionospheric irregularities [2]. Thus, there is good reason to expect obliqueincidence $E$ s propagation to obey the secant law.

\section{Experimental Results}

In 1951 and 1952 the National Bureau of Standards operated a pulsed, sweep-frequency, oblique-incidence, ionosphere sounding experiment between Sterling, Virginia, and St. Louis, Missouri $[3,4,5]$.

1 Contribution from Central Radio Propagation Laboratory, National Bureau of Standards, Boulder, Colo.

2 Figures in brackets indicate the literature references at the end of this paper.
Vertical soundings were obtained simultaneously at the midpoint of this $1,150-\mathrm{km}$ path. One-hop sporadic $E$ transmission was frequently observed over this circuit, thereby providing an opportunity to examine the relation between the highest frequencies of reflection observable at oblique and vertical incidence. A preliminary account of the results was included by Smith [6].

Two 24-hr periods were studied for this purpose, providing 123 cases of essentially simultaneous observations of $E$ s reflection at vertical and oblique incidence. The ratio of the top frequency observed over the oblique path to that at vertical incidence at the midpoint is shown in figure 1 as a function of $E_{\mathrm{s}}$ virtual height. The cases in which multiple echoes at vertical incidence had the same highest frequency are indicated on the graphs in order to identify instances of power independence and high reflectivity of the $E$ s. The full curve is the secant $\Phi$ relationship, plotted versus the height of the midpoint of the triangular path.

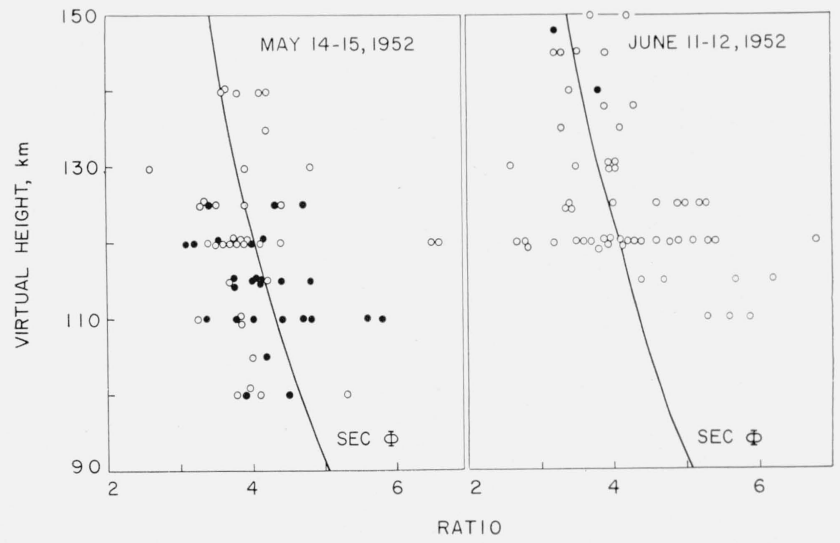

FIGURE 1. Distribution of ratio of oblique to vertical-incidence sporadic E top frequency with virtual height.

denotes points for which vertical-incidence multiples extend to same frequency. 
The experimental ratios, $f_{\mathrm{ob} 1} / f_{v}$ are seen to be in the same range as sec $\Phi$. Whether there is a significant variation of the ratios with height corresponding to sec $\Phi$ is a subject for a more detailed analysis. These results, however, lend support to the significance of $f E_{\mathrm{s}}$, the vertical-incidence $E_{\mathrm{s}}$ top frequency, as a parameter useful in propagation studies.

\section{References}

[1] J. A. Thomas and E. K. Smith, A survey of the present knowledge of sporadic $E$ ionization, J. Atmospheric and Terrest. Phys. 13, 295, (1959)

[2] E. V. Appleton and W. J. G. Beynon, An ionospheric attenuation equivalence theorem, J. Atmospheric and Terrest. Phys. 6, 141 (1955).
[3] P. G. Sulzer and E. E. Ferguson, Sweep-frequency obliqueincidence ionospheric measurements over an $1,150-\mathrm{km}$ path, Proc. IRE 40, 1124 (1952).

[4] Bernard Wieder, Some results of a sweep-frequency propagation experiment over an $1,150-\mathrm{km}$ east-west path, J. Geophys. Research 60, 395 (1955).

[5] V. Agy and K. Davies, Ionospheric investigations using the sweep-frequency pulse technique at oblique incidence, J. Research NBS 63D, 151 (1959).

[6] E. K. Smith, Worldwide occurrence of sporadic E, NBS Circ. 582, 227 (1957). 